\title{
Treatment of primary pulmonary hypertension with continuous intravenous prostacyclin
}

Primary pulmonary hypertension (unexplained or idiopathic pulmonary hypertension) is a rare disease of the pulmonary vasculature that primarily affects young adults who are otherwise healthy, and usually runs a rapidly fatal course. Although Romberg published the first report of a patient with unexplained pulmonary hypertension in $1891^{1}$ and Dresdale described the entity as primary pulmonary hypertension in $1951,{ }^{2}$ the pathogenesis of this disease remains unknown. Endothelial injury, pulmonary vasoconstriction, and in situ thrombosis have been proposed as causative factors.

In the National Institute of Health Registry on Primary Pulmonary Hypertension, 194 patients were diagnosed between 1981 and 1985 and followed through 1988. The median survival was $2 \cdot 8$ years after diagnosis with a five year survival rate of $34 \% .^{3}$ Although no known cure exists, treatment for the disease has significantly improved over the past decade.

\begin{abstract}
Medical treatment
Conventional medical approaches include treatment with anticoagulants and oral vasodilators. Nevertheless, many patients are refractory to conventional medical treatment and require transplantation (lung or heart/lung). In these patients, the waiting time for transplantation often exceeds their expected survival. This led to our evaluating the effects of continuous intravenous prostacyclin in patients unresponsive to oral vasodilator treatment, initially as a bridge to transplantation, and subsequently as an alternative treatment to transplantation in selected patients. These studies demonstrated improved survival as well as improved haemodynamics and quality of life in children and adults treated with chronic prostacyclin. ${ }^{4-6}$ Despite the demonstrated significant improvement with chronic prostacyclin treatment, we do not recommend long term prostacyclin treatment for all patients with primary pulmonary hypertension based on risk:benefit considerations. Currently, our goal with medical treatment, which includes optimal vasodilator treatment, is to defer transplantation until clinically indicated, as well as to avoid transplantation in selected circumstances as long as medical treatment is providing improved quality of life and survival.
\end{abstract}

\section{Anticoagulant treatment}

The presence of endothelial injury in the pulmonary vascular bed in conjunction with a low cardiac output makes these patients prone to develop pulmonary thrombi. In 1984, Fuster reported in a retrospective study that survival was better in 78 patients who received oral anticoagulants than in 37 patients who were not anticoagulated. ${ }^{7}$ Rich subsequently confirmed the effect of anticoagulation on survival in primary pulmonary hypertension in a prospective study. ${ }^{8}$ The enhanced risk of intravascular thrombosis explains the valuable role of long term anticoagulants in the treatment of primary pulmonary hypertension. Based on these two studies, we now recommend chronic anticoagulation with warfarin for all patients with primary pulmonary hypertension unless there is an absolute contraindication.

\section{Oral vasodilator treatment}

Our current approach to treatment of primary pulmonary hypertension includes oral vasodilator therapy (primarily with calcium channel blockers), continuous intravenous prostacyclin, or both. Acute vasodilator drug testing has been shown to predict long term prognosis with chronic oral vasodilator treatment. ${ }^{8}$ Patients with a positive acute response, defined as a $20 \%$ or greater decrease in mean pulmonary artery pressure with no change or an increase in cardiac index during acute vasodilator testing, ${ }^{9}$ have a significantly improved five year survival on chronic oral vasodilator treatment compared with nonresponders $(94 \%$ $v 42 \%){ }^{8}$ Unfortunately, in our experience, only $12 \%$ of adult patients have a positive response with acute vasodilator testing. In children, the response rate is greater $(41 \%)$ with the acute responders having a significantly improved five year survival on chronic oral vasodilator treatment compared with nonresponders (97\% $v 39 \%)$. Nonetheless, only $10 \%$ to $25 \%$ of adults and about $40 \%$ of children improve clinically and live longer when treated with chronic oral vasodilator treatment. Until recently, all patients unresponsive to oral vasodilator therapy have required transplantation but $30 \%$ to $40 \%$ died awaiting the treatment. Chronic calcium channel blockade treatment is contraindicated in patients with severe right heart failure (mean right atrial pressure $20 \mathrm{~mm} \mathrm{Hg}$ or higher); it also needs to be evaluated under invasive monitoring in patients with a mean right atrial pressure of less than $20 \mathrm{~mm} \mathrm{Hg}$ to prevent untoward adverse effects with this treatment.

\section{Prostacyclin treatment}

Prostacyclin is a potent short acting vasodilator and inhibitor of platelet aggregation that is produced by the vascular endothelium. Prostacyclin decreases pulmonary vascular resistance and increases cardiac output and systemic oxygen delivery when administered acutely to patients with primary pulmonary hypertension. ${ }^{10}$

Higenbottam was the first to use continuous prostacyclin infusion in a patient with severe primary pulmonary hypertension in whom he demonstrated remarkable improvement. ${ }^{11} \mathrm{He}$ subsequently reported his initial experience with 10 patients in whom the majority responded well to prostacyclin treatment with significant improvement in quality of life and exercise capacity. ${ }^{12}$ In 1993 he presented his experience with 25 patients treated with prostacyclin while awaiting transplantation and 19 patients listed for transplantation who did not receive prostacyclin. ${ }^{13}$ This observational study suggested that prostacyclin improved the likelihood of survival to transplantation. 
In 1987, we began randomised, multicentre clinical trials with chronic intravenous infusions of prostacyclin for patients who failed conventional medical treatment. In 1994, we reported improved survival in 17 patients who had failed conventional medical treatment and were treated with continuous intravenous prostacyclin in addition to conventional treatment. ${ }^{5}$ We compared the prostacyclin treated patients with historical controls from the National Institute of Health Registry on Primary Pulmonary Hypertension. The one, three, and five year survival rates for the prostacyclin treated patients were $87 \%, 63 \%$, and $54 \%$, respectively, compared with $77 \%$, $41 \%$, and $27 \%$ for the NIH registry patients. Subsequently, a 12 week randomised multicentre study evaluated the effects of prostacyclin and conventional treatment versus conventional treatment alone in 81 patients with severe primary pulmonary hypertension. ${ }^{6}$ This is the first randomised study to demonstrate clinical and haemodynamic improvement as well as increased survival in prostacyclin treated patients compared with conventional treatment alone. Based on this study, prostacyclin has been approved by the United States Food and Drug Administration as treatment for severe primary pulmonary hypertension. Long term follow up of 357 patients treated with chronic prostacyclin has confirmed the improved survival seen in the 12 week study. We therefore recommend consideration of continuous intravenous prostacyclin for patients with primary pulmonary hypertension who are unresponsive to oral vasodilator treatment.

In addition to improved survival, quality of life was improved dramatically in many of the patients treated with long term prostacyclin. Although all patients have a central venous line and are on continuous intravenous prostacyclin 24 hours a day, many patients have returned to work or school and lead an active life.

Despite our success in treating patients with end stage primary pulmonary hypertension with prostacyclin, many questions remain unanswered. The clinical and haemodynamic improvements cannot always be predicted by the patients' acute response to initial prostacyclin testing. Not uncommonly, despite a patient having no haemodynamic improvement acutely, chronic prostacyclin treatment results in marked haemodynamic improvement at follow up evaluation. This improvement with chronic prostacyclin treatment in patients who have not responded in the short term has not been seen with chronic oral vasodilator treatment. These observations suggest that prostacyclin may have significant effects on remodelling of the pulmonary vascular bed in addition to its known effects as a potent pulmonary vasodilator and inhibitor of platelet aggregation. The implication is that part of the increase in pulmonary vascular resistance previously considered to be fixed might be reversible.

Minor complications related to the use of prostacyclin occur frequently and include jaw pain, diarrhoea, flushing, headaches, nausea, and vomiting. Serious complications are most often due to the delivery system and include catheter related sepsis and thrombotic events. Malfunctions of the drug delivery system-including occlusions, perforations, and dislodgments of the catheter, and pump malfunction-have resulted in temporary interruption of the infusion. Although it has been well tolerated in some patients, temporary interruption has precipitated the sudden onset of increased symptoms such as syncope, and on rare occasions has resulted in death from an acute pulmonary hypertensive crisis. Additional problems related to the delivery system include irritation, infection, bleeding, and pain at the catheter site. Despite the complexity of the drug delivery system, children as young as 2 months and adults older than 70 have successfully been started on continuous intravenous prostacyclin. Although we have seen some patients whose pulmonary artery pressures have decreased from suprasystemic levels to normal on long term prostacyclin treatment, we do not believe that chronic prostacyclin has "cured" the disease; rather, treatment appears to be successfully "palliating" the disease process. Currently, patients who are started on intravenous prostacyclin are begun with the anticipated plan to continue until transplantation or death. To date, despite significant improvements on chronic prostacyclin, up to nine years in the United States and 12 years in the United Kingdom, no patient who has improved on prostacyclin has been taken off treatment. Whether in the future, as we gain more insight into the pathogenesis and pathophysiology of primary pulmonary hypertension, we will be able to discontinue prostacyclin in selected patients remains unknown. It is exceedingly important for physicians and patients to understand that when we initiate chronic prostacyclin treatment, we are doing so anticipating treatment on an indefinite basis.

Chronic prostacyclin may not only help to stabilise the disease before suitable donor organs are available but may also enhance the likelihood of a successful transplantation. Pasque recently reported the results of single lung transplantation for primary pulmonary hypertension. ${ }^{14}$ The actuarial survival for 24 patients at one, two, and three years were $87 \%, 76 \%$, and $68 \%$, respectively, which is not significantly different from our experience with 357 patients treated with prostacyclin in whom the survival rates were $81 \%, 72 \%$, and $63 \%$. These data demonstrate that both continuous intravenous prostacyclin and single lung transplantation improve survival compared with conventional medical treatment alone.

\section{Atrial septostomy}

An additional therapeutic intervention that may be useful in sustaining seriously ill patients awaiting transplantation is a palliative blade balloon atrial septostomy. A small atrial septal defect has been created in patients with recurrent syncope or near syncope as well as significant right heart failure despite optimal medical treatment including continuous intravenous prostacyclin. In our experience, in selected patients, transplantation can be deferred for several years because of prolonged survival with an improved quality of life following blade balloon atrial septostomy. ${ }^{15}$

\section{Summary}

Our ultimate goal in treating patients is to improve their quality of life and to increase survival. The optimal treatment for primary pulmonary hypertension will continue to change as our understanding of its causes improves and as progress is made in lung transplantation. There is no one best treatment for all patients. Optimal medical and surgical treatment must be tailored to the individual with changes in therapeutic regimens based on serial evaluations. Quality of life and survival have improved with current treatments and the future should offer additional therapies-inhaled nitric oxide, endothelin receptor blockers, and other modulators of the pulmonary vascular bed-to improve further the treatment of this disease.

In conclusion, although primary pulmonary hypertension, if untreated, is most often a rapidly progressive and fatal disease, recent advances in the treatment have significantly improved the outcome for patients. Although transplantation is often considered the only definitive treatment for patients with primary pulmonary hypertension, medical treatment seems to be an effective long term 
palliation to successful transplantation as well as a possible alternative treatment to transplantation in selected children and adults. Quality of life and cost analyses, as well as longer follow up studies are needed to determine the best treatment for patients with primary pulmonary hypertension.

ROBYN J BARST

Division of Pediatric Cardiology,

Columbia University College of Physicians and Surgeons,

3959 Broadway BH 262N,

New York, NY 10032, USA

1 Romberg E. Uver sklerose der lungenarterien. Dtsch Arch Klin Med 1891; 48:197.

Dresdale DT, Schultz M, Michtom RJ. Primary pulmonary hypertension. Am $\mathcal{F}$ Med 1951;11:686-705.

3 D'Alonzo GE, Barst RJ, Ayres SM, Bergofsky EH, Brundage BH, Detre

KM, et al. Survival in patients with primary pulmonary hypertension: KM, et al. Survival in patients with primary pulmonary hypertension: results

4 Rubin LJ, Mendoza J, Hood M, McGoon M, Barst RJ, Williams WB, et al. Treatment of primary pulmonary hypertension with continuous intravenous prostacyclin (epoprostenol): results of a randomised trial. Ann Intern Med 1994;112:485-91.

5 Barst RJ, Rubin LJ, McGoon MD, Caldwell EJ, Long WA, Levy PS. Survival in primary pulmonary hypertension with long-term continuous intravenous prostacyclin. Ann Intern Med 1994;121:409-15.
6 Barst RJ, Rubin LJ, Long WA, McGoon MD, Rich S, Badesch DB, et al. A comparison of continuous intravenous epoprostenol (prostacyclin) with comparison of continuous intravenous epoprostenol (prostacyclin) with Med 1996;334:296-301.

7 Fuster V, Steele PM, Edwards WD, Gersh BJ, McGoon MD, Frye RL. Primary pulmonary hypertension: natural history and the importance of thrombosis. Circulation 1984;70:580-7.

8 Rich S, Kaufmann E, Levy PS. The effect of high doses of calcium-channe blockers on survival in primary pulmonary hypertension. $N \mathrm{Engl} f \mathrm{Med}$ 1992;327:76-81.

9 Barst RJ. Pharmacologically induced pulmonary vasodilatation in children and young adults with primary pulmonary hypertension. Chest 1986 89:497-503.

10 Moncada S, Vane RJ. Arachidoic acid metabolites and the interactions between platelets and blood-vessel walls. $N$ Engl $\mathcal{f}$ Med 1979;300: $1142-7$.

11 Higenbottam TW, Wheeldon D, Wells FC, Wallwork J. Long-term treatment of primary pulmonary hypertension with continuous intravenous ment of primary pulmonary hypertension with con

12 Jones DK, Higenbottam TW, Wallwork J. Treatment of primary pulmonary hypertension with intravenous epoprostenol (prostacyclin). $B r$ monary hypertension with

13 Higenbottam TW, Spiegelhalter D, Scott JP, Fuster V, Dinh-Xuan AT Caine $\mathrm{N}$, et al. Prostacyclin (epoprostenol) and heart-lung transplantaCaine $\mathrm{N}$, et al. Prostacyclin (epoprostenol) and heart-lung transplanta-
tion as treatments for severe pulmonary hypertension. Br Heart $f$ 1993; 70:366-70.

14 Pasque MK, Trulock EP, Cooper JD, Triantafilou AN, Huddelston CB, Rosenbloom $\mathrm{M}$, et al. Single lung transplantation for pulmonary hypertension. Circulation 1995;92:2252-8.

15 Kerstein D, Levy PS, Hsu DT, Hordorf AJ, Gersony WM, Barst RJ. Blade balloon atrial septostomy in patients with severe primary pulmonary hypertension. Circulation 1995;91:2028-35.

\section{STAMPS IN CARDIOLOGY}

\section{Ferdinand Sauerbruch (1875-1951)}

Germany (West Berlin) issued this single 50 pfennig stamp in 1975 to commemorate the centenary of the birth of Sauerbruch. Other medical personalities occasionally featured on stamps from West Berlin have included Virchow (1952), von Humboldt (1952, 1985), Koch (1960), and von Graefe (1978).

Ferdinand Sauerbruch was a great pioneer of both thoracic and cardiac surgery who worked first in Zurich and Munich and then from 1928 at the Charité Hospital in Berlin. $\mathrm{He}$ invented inflow occlusion of the heart as a way of making it easier to suture cardiac lacerations. His method, described in 1907, was to compress the base of the heart and the venae cavae with two fingers of the same hand, or to press on the right heart with the thumb and finger. In 1912 he performed pulmonary embolectomy using the method described in 1908 by Trendelenburg. As was usual with this operation, which was still being done 20 years later, his patient died. In 1913 he was the first surgeon to resect the pericardium for constrictive pericarditis and, despite his concern that he had freed only the right ventricle, his patient was well 11 years later. In 1931 he became the first to operate, albeit by mistake, on a cardiac aneurysm. The patient had a mediastinal mass which spurted blood into his face when it was incised. He inserted a finger and found a hole surrounded by a resilient ring beyond which was a cardiac chamber which seemed to be the right ventricle. He closed the hole with silk sutures "tying knots we could not see". The patient made a complete recovery. But perhaps he is best known for the remarkable differential pressure chamber which he devised in 1905 and which permitted the thoracic cavity to be opened without the lung collapsing. Initially his chief, Professor

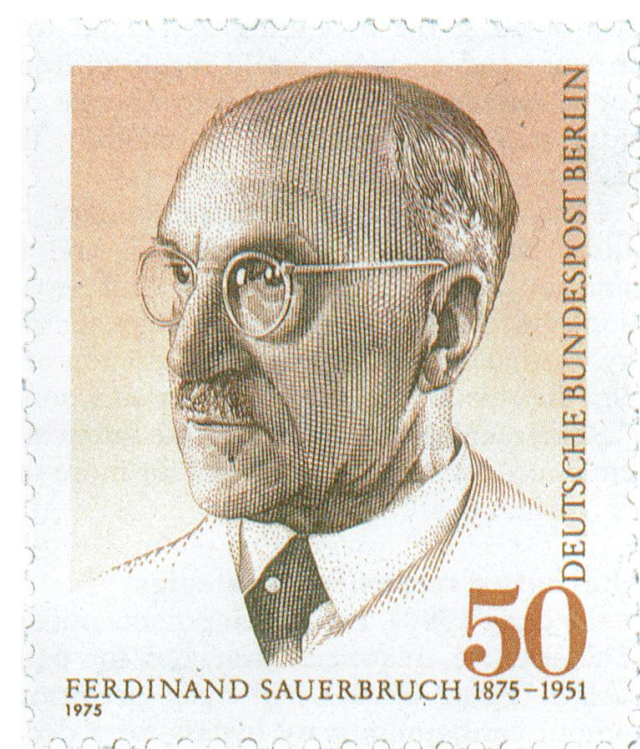

von Mikulicz, called him a charlatan, but later he fully supported his work. Laurence O'Shaughnessy, the British pioneer of cardiac revascularisation studied under him. Sauerbruch's autobiography in 1953, $A$ Surgeon's Life, reveals a dynamic man, with deep concerns for his patients and colleagues. It is full of interesting stories ranging from a chest operation on the daughter of Lord Cavendish-Bentinck, removing a tooth from the young Lenin, who could not afford a dentist, to exciting Hitler's jealousy by making friends with the dog Hitler thought was devoted to him. 